\title{
Análisis
}

\section{Libros electrónicos en la Red de Bibliotecas del CSIC. Creación de una colección común}

\author{
Por Mercedes Baquero-Arribas
}

\begin{abstract}
Resumen: La colección de recursos digitales de la Red de Bibliotecas del CSIC ha estado formada fundamentalmente por revistas electrónicas. Pero en los últimos 3 años el modelo de adquisición, gestión y accesibilidad aplicado a las revistas se ha exportado a los libros electrónicos. La primera fase de la introducción de libros electrónicos en el CSIC no siguió una estrategia definida. Desde 2007 se ha establecido un plan de cooperación dentro de la propia Red que persigue crear una colección común de libros electrónicos de calidad. Se presenta la experiencia de compras cooperativas entre las bibliotecas de la Red, las colecciones seleccionadas, cómo se hacen accesibles a los usuarios, el uso que han tenido, las inversiones realizadas y los planes futuros de consolidación de los libros electrónicos en el CSIC.
\end{abstract}

Palabras clave: Libros electrónicos, Consejo Superior de Investigaciones Científicas, CSIC, Adquisición cooperativa, Colección digital, Red de bibliotecas.

Title: Ebooks in the CSIC Library Network. Building a common collection

Abstract: The collection of digital resources of the CSIC Library Network has been developed mainly for electronic journals. However in the last three years its model for acquisition, management and accessibility applied to journals has been tried to ebooks. The first phase of the introduction of electronic books in the CSIC did not follow a defined strategy. Since 2007 a plan of cooperation has been established within the network in order to create a common collection of quality electronic books. In this article we describe the experience of cooperative purchasing among libraries in the network, the selected collections, how to make them accessible to library patrons, their usage up to now, the investments made and future plans to consolidate the CSIC ebooks collection.

Keywords: Ebooks, Spanish National Research Council, CSIC, Cooperative acquisition, Digital library collection, Library network.

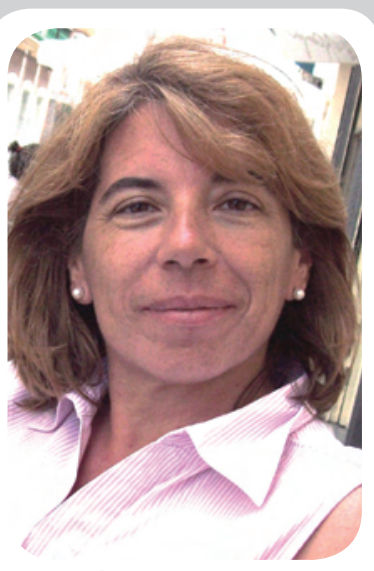

Mercedes Baquero-Arribas es licenciada en filología hispánica por la Universidad Autónoma de Madrid. Titulado superior especializado del Consejo Superior de Investigaciones Científicas (CSIC), donde es responsable de la gestión de la colección impresa y digital de la Red de Bibliotecas, desde la Unidad de Coordinación de Bibliotecas.

Baquero-Arribas, Mercedes. "Libros electrónicos en la Red de Bibliotecas del CSIC. Creación de una colección común". El profesional de la información, 2010, septiembre-octubre, v. 19, n. 5, pp. 477-485.

DOI: 10.3145/epi.2010.sep.05

LOS LIBROS ELECTRÓNICOS se han ido introduciendo en las bibliotecas universitarias y científicas españolas a un ritmo lento. Después de un período inicial de observación sobre el nuevo soporte, a principios de esta década, terminamos 2010 habiendo adquirido en las bibliotecas colecciones de ebooks, por varios procedimientos y de un modo ya menos inseguro.

El presente artículo describe la experiencia de la Red de Bibliotecas del Consejo Superior de Investigaciones Científicas en la adquisición centralizada de libros electrónicos para su comunidad. Experiencia que tiene una evolución singular porque se apoya en el trabajo previo hecho sobre las revistas electrónicas; se basa específicamente en el entorno del libro científico; ya puede exponer resultados sobre las adquisiciones hechas y, en última instancia, por tratarse el CSIC de un organismo con unas características únicas en España.

La bibliografía profesional sobre el libro electrónico dedica numerosas páginas a aclarar el término (si se habla del dispositivo físico que sirve para leer o del contenido legible); analiza las cualidades de la tinta electrónica; estudia la viabilidad o no de descargar un libro completo o sólo un capítulo; analiza si se puede considerar libro electrónico la digitalización de otro impreso; dentro de las bibliotecas, hablan de cómo prestar los ebooks. En la prensa general aparecen también a menudo artículos sobre el futuro del libro electrónico, si suplantará al impreso o convivirá con él.

Todos estos enfoques complementan la visión del libro electró- 
nico, pero no son lo fundamental cuando de lo que nos ocupamos es de la monografía científica, y nos interesa observar su aceptación en bibliotecas de investigación como las del CSIC.

Los usuarios de estos libros científicos aprecian en ellos las mismas prestaciones y funcionalidades a las que están acostumbrados desde hace casi una década en las revistas electrónicas. Quieren lo antes posible la monografía que les interesa. Apuestan por las publicaciones más recientes. Leen una parte del libro (uno o varios capítulos) más que el libro completo (como leen uno o varios artículos de una revista, no el fascículo completo de una vez). Quieren encontrarlo en su biblioteca virtual que está abierta permanentemente. Quieren consultarlo desde fuera de su despacho o laboratorio. Y no quieren que haya restricciones de uso sobre ellos. Cuantos más libros electrónicos se ponen a su disposición, más los usan.

\section{"Cuantos más libros electrónicos se ponen a disposición de los investigadores, más los usan"}

Aunque la demanda de libro impreso por parte de los investigadores del CSIC no ha decaído, sus bibliotecas se han comprometido con el nuevo formato, destinando parte de sus presupuestos individuales, cada año más, a la compra cooperativa de libros electrónicos, tal y como se describe en este trabajo.

\section{Proceso de adquisición de recursos digitales para la Biblioteca virtual del CSIC}

Desde 2001 el CSIC ha centralizado la adquisición de sus recursos de información científica.
La Unidad de Coordinación de Bibliotecas (UCB), oficina gestora de la Red de Bibliotecas del CSIC, es la encargada de esa adquisición. Para ello, en 2001 centralizó los presupuestos que las bibliotecas recibían para la suscripción de publicaciones periódicas, y también se hizo cargo de todas las revistas que por separado suscribía cada una de las bibliotecas. Centralizó igualmente la adquisición del conjunto de bases de datos de información científica que hasta entonces se suscribían por diversas vías. El resultado, especialmente importante en el caso de las publicaciones periódicas fue tener por primera vez un escenario comprensible, completo e integrado de en qué estaban gastando sus presupuestos las bibliotecas y, por la misma vía, qué editores y títulos son los que los científicos de la institución querían que ésta comprara. Este escenario -en aquel tiempo mayoritariamente impreso- orientó la selección de revistas electrónicas por parte de la $U C B$ a lo largo de los siguientes 8 años. El objetivo perseguido: crear una colección digital común.

Se centralizaron los aspectos económicos de la adquisición y, al mismo tiempo, los de gestión de la colección resultante, que se hace accesible a la comunidad mediante unas herramientas que persiguen la integración y la máxima accesibili$\operatorname{dad}($ Metalib $+S F X+P A P I)$.

El proceso se lleva a cabo dentro de la línea de trabajo que la $U C B$ presenta a las autoridades del $C S I C$, y que éstas asumen y respaldan, convirtiéndola en "política institucional". Desde 2001 el objetivo es el descenso de la colección impresa, aumento de la digital, pero contención del gasto. Se mantiene esta línea en el actual Plan estratégico 2010-2013.

Es necesario apuntar la extraordinaria complejidad del CSIC en cuanto institución: más de 120 centros de investigación, de todos los tamaños, a los que dar servicios bibliotecarios. Una red de 70 bibliotecas científicas (es decir, en 50 institutos o unidades de investigación no hay bibliotecas físicas, pero sí Biblioteca virtual). Se investiga sobre todos los campos del conocimiento, organizados en 8 áreas científicas (ciencia y tecnologías físicas, químicas, de materiales, de alimentos, biología y biomedicina, ciencias agrarias, recursos naturales, humanidades y ciencias sociales). El número de investigadores de plantilla se acerca a los 3.500. No hay estudiantes. El grueso de los recursos digitales está contratado para todos los centros, pero hay una parte significativa que se contrata ya sea para un área concreta, o para un grupo de centros que anteriormente tuviera suscripción impresa a ese editor. Son contenidos actuales, pero de algunos editores ha sido posible adquirir archivos retrospectivos. Una preocupación desde el primer momento ha sido garantizar a los científicos el acceso a los recursos cuando estuvieran fuera del campus (resuelto gracias al servicio de autenticación $P A P I)$.

Esta organización y estrategia para la creación de la colección digital de la Red de Bibliotecas se ha mostrado muy eficiente, y en la actualidad tenemos una de las bibliotecas virtuales más importantes de España en cuanto a contenidos, calidad y accesibilidad. Todo lo descrito hasta ahora ha afectado a las bases de datos y a las publicaciones periódicas. Las primeras, especialmente en los últimos años, están experimentando un descenso de uso que provoca su evaluación constante y una disminución en el número suscrito. En lo que se refiere a las publicaciones periódicas, se ha llevado a cabo una sistemática y minuciosa transición del soporte papel al digital, eliminando las suscripciones duplicadas y apostando, siempre que económica y conceptualmente ha 


\section{PAP I}

Servicio desarrollado por Redlris que proporciona acceso a cualquier tipo de información electrónica mediante mecanismos de identificación (nombre de usuario y contraseña, firma electrónica, certificados, etc.) de forma que el acceso se hace independientemente de la dirección IP del ordenador desde el que se conecta.

En el CSIC, la identificación del usuario se realiza a través de la intranet institucional, pero PAPI resuelve dos problemas:

1. El acceso de los investigadores desde lugares distintos a sus puestos habituales de trabajo (accesos "off-campus"), ya sea por desplazamientos temporales o por trabajar desde casa o desde otras instituciones.

2. El acceso de usuarios desde centros mixtos y centros propios que utilizan direcciones IP que no pertenecen a redes de comunicación del CSIC sino a redes de otras instituciones, en general de las universidades.

PAPI realiza funciones similares a Athens o Shibboleth.

sido posible, por la contratación de licencias institucionales para todo el CSIC, primero de los grandes editores científicos, más adelante de publicaciones de sociedades y grupos más pequeños, o incluso de títulos individuales.

\section{"La Unidad de Coordinación ha optado por la compra de libros electrónicos y no por la suscripción"}

A partir de 2007 el modelo de adquisición y gestión de recursos digitales se ha querido exportar progresivamente al libro electrónico. ¿Por qué no se hizo en los mismos años que se estaba operando sobre las bases de datos y las publicaciones periódicas? Hay dos razones de peso:

- la oferta editorial de libros electrónicos anterior a ese año 2007 no era aún suficiente como para hacer una apuesta decidida hacia este soporte;
- no ha habido centralización del presupuesto para adquisiciones de monografías.

\section{Características \\ de la compra de libros electrónicos}

La $U C B$ ha optado, como la mayoría de las bibliotecas universitarias y los consorcios, por la compra y no por la suscripción. Se sigue así la propia rutina de la adquisición de monografías en el entorno papel que (no siendo series o standing orders) se compran, no se suscriben. Se integran en los fondos de la biblioteca para siempre, y no se contrae el compromiso económico de volver a pagar al año siguiente.

No se ha experimentado en la $U C B$, pero en el mercado existe un modelo de compra llamado "por evidencia", que consiste en disponer de una colección completa durante un período de tiempo, y finalmente elegir sólo un conjunto de libros para la compra definitiva en función del uso).

La compra puede hacerse además en cualquier momento del año, no como las suscripciones de publi- caciones periódicas, que imponen trabajar de un año para el siguiente. Además, de momento, debido a que no se alcanzan los topes que la Ley de contratos del sector público obliga para celebrar concursos -ya que cada biblioteca recibe la factura de lo que ella aporta a la compra general- es innecesario un procedimiento que en el caso de la revistas digitales es ineludible.

Puesto que no ha habido un presupuesto centralizado para las monografías la tarea de la $U C B$ ha sido "concienciar" a las bibliotecas de la Red de que colaborando entre todas con parte de sus presupuestos individuales se conseguiría una colección común llena de ventajas.

La selección de qué libros comprar se ha hecho pidiendo ofertas a los editores con los que se trabaja habitualmente en revistas, ofertas diseñadas para el CSIC (contenidos que sean de interés en las áreas de investigación) y que han sido evaluadas por las bibliotecas y sus usuarios.

Para orientar la selección de recursos, así como para las revistas se contó con la información recogida al centralizar la gestión de suscripciones en 2001, para los libros no se tiene el mismo instrumento. El catálogo colectivo es un referente parecido, pero no igual, ya que muchas de las obras catalogadas no han ingresado por compra. Por otra parte, está claro que la intención no es convertir los fondos impresos ya existentes en algo digital (como pudo ser con las revistas) sino que siempre que una monografía esté en formato papel y electrónico, la opción de compra sea por este último.

A diferencia de la suscripción de publicaciones periódicas, en las que el volumen de títulos duplicados en el CSIC ha sido un pesado lastre que los editores han hecho pagar muy caro en la transición a 
la revista digital, en el caso de las monografías la colección impresa no supone ningún condicionante. $Y$ el modelo de compra que proponen los editores (al CSIC y a cualquier institución) tiene en cuenta la cantidad de usuarios potenciales de la misma, no el gasto previo que esta hubiera hecho en soporte papel.

\section{Ventajas de la adquisición en común}

El objetivo es crear entre todos, para el CSIC, una colección de base de libros electrónicos de calidad. Para ello y con el fin de animar a las bibliotecas a cooperar en la adquisición centralizada, el acceso a los ebooks será para toda la institución y no, como en las compras individuales, únicamente para el centro que los adquiere.

Se ofrece también la catalogación de los libros en la propia $U C B$ (cargas de registros marc para la mayoría de las colecciones, aunque algunas otras han sido catalogadas por el propio departamento de proceso bibliográfico de la $U C B$ ). Igualmente, la tediosa activación de los libros, el envío de IPs a los editores, la supervisión y firma de licencias, el acceso mediante el servicio PAPI de autenticación, son tareas de las que se ven descargadas las bibliotecas si apuestan por esta compra colectiva.

Pero la más clara ventaja es comprender que los pequeños presupuestos de las bibliotecas por separado hacen muy poco mientras que juntos se pueden adquirir más y mejores colecciones, y hay garantías de que no se comprarán obras duplicadas.

Lo que se presenta como ventajas ofrecidas a las bibliotecas son a su vez requisitos que la $U C B$ exige a los editores: acceso a toda la institución, licencias bajo el modelo de compra de libros, entrega de registros marc y de datos de uso (en formato Counter siempre que sea posible), productos ajustados a estándares que permitan su inclusión en la Biblioteca virtual.

En cuanto a la facturación, mientras no exista centralización del presupuesto la $U C B$ intermedia en la tramitación de facturas entre el editor o agente y el centro.

\section{"Los presupuestos de las bibliotecas por separado hacen muy poco mientras que juntos se pueden adquirir más y mejores colecciones"}

\section{Introducción de libros electrónicos. Período inicial 2004-2006}

La colección común de libros electrónicos tiene una fase previa a las compras centralizadas que se lleva a cabo entre 2004 y 2006. En estas fechas se incorporan:

- 2004: Current protocols (Wiley) y Early English books online (EEBO)

- 2005: Elsevier book series y E-libro (Ebrary para España y Portugal)

- 2006: Elsevier reference works

Entran en la Biblioteca virtual por diversas vías, sin responder a una estrategia definida de creación de colección común. Tanto los $\mathrm{Cu}$ rrent protocols como EEBO han sido inicialmente suscritos por centros individuales (Centro Nacional de Biotecnología en el primer caso, Instituto de Filología en el segundo) con sus propios presupuestos. La $U C B$ gestiona la ampliación de la licencia individual de los $\mathrm{Cu}$ rrent protocols para que sea de acceso institucional, y en el caso de $E E B O$ contribuye con el centro a un pago aplazado en varios años, que finalmente convierte la sus- cripción en una compra definitiva para la institución. En esos años el horizonte de los libros electrónicos no estaba tan definido como lo empieza a estar ahora, e incluso no todos los bibliotecarios consideran estos productos (en un caso manuales de laboratorio, en otros libros impresos digitalizados) libros electrónicos. Por lo que respecta a la historia de la Biblioteca virtual, sí son los primeros ebooks que se incorporaron.

Las colecciones de Elsevier de aquellos años, Book series y Reference works fueron complementos de las suscripciones que se hicieron a su producto ScienceDirect. Pero, aun no siendo su suscripción una decisión de la Red de Bibliotecas, sí han sido muy apreciadas por los usuarios. Su uso (lo veremos más adelante) es significativo. Tanto, que una de las últimas decisiones ha sido dar a las Book series más relevancia en el catálogo, mediante la catalogación individual de los volúmenes que las componen (es decir, darles categoría de libro electrónico, antes que de publicación periódica).

\section{Compras cooperativas 2007-2009}

En esta fase sí se entra de lleno en la compra coordinada entre todas las bibliotecas del CSIC, siguiendo el modelo descrito (desde la $U C B$ se han pedido ofertas a los editores, que se dan a conocer a las bibliotecas y a los usuarios, y se sugiere una adquisición compartida, insistiendo en las ventajas). El resultado en estos años ha sido:

2007. Entre 8 bibliotecas:

- 2 colecciones de Elsevier

- colección de arquitectura de Springer

Además, algunas bibliotecas han ido comprando individualmente colecciones de libros electrónicos que no son accesibles a todo el Consejo. 
2008. Entre 10 bibliotecas:

- 3 colecciones de Elsevier

- títulos sueltos de Wiley

- 3 colecciones de Springer (ampliación de licencias individuales a licencias institucionales).

2009. Entre 30 bibliotecas:

- 3 colecciones de Ebrary

- Elsevier: nuevos años de las colecciones ya disponibles

- 2 colecciones nuevas de Springer, y períodos nuevos de las 3 anteriores

- más títulos sueltos de Wiley, y del agregador MyiLibrary

- E. J. Brill, todos sus libros publicados en 2009

- De Gruyter, todos sus libros publicados en 2009

Aunque éstas son las colecciones y editoriales que han interesado, los editores evaluados por las bibliotecas han sido más, y en el futuro podrían formar parte de la colección digital: Emerald, Gale Virtual Library, Cambridge University Press, Oxford University Press, Palgrave, Taylor \& Francis.

En 2009 se presentó ante los coordinadores de las áreas científicas del CSIC la iniciativa de la adquisición cooperativa, para procurar su implicación en las propuestas de contenidos, y también se recuperaron para la inversión en libros digitales las cantidades que unos pocos centros sin biblioteca recibían para adquisición de monografías. Los libros impresos que llegan a un centro sin biblioteca física ni personal bibliotecario que los incorpore al catálogo, se pierden para el resto de los potenciales usuarios, pero no sucede así si se convierten en parte de la Biblioteca virtual.

\section{Colección de libros electrónicos}

En junio de 2010 superaba los 208.500 títulos. Los principales productos se muestran en la tabla 1.

\begin{tabular}{|l|r|}
\hline EEBO & 125.000 \\
\hline E-libro & 74.000 \\
\hline Springer e-books & 7.000 \\
\hline Elsevier e-books & 1.500 \\
\hline Wiley e-books & 400 \\
\hline E. J. Brill & 304 \\
\hline De Gruyter & 275 \\
\hline MyiLibrary & 112 \\
\hline Current protocols & 8 \\
\hline Total & 208.599 \\
\hline
\end{tabular}

\section{¿Cómo se dan a conocer?}

La explicación de qué colecciones hay, número de títulos y centros con acceso está en la web de la Red de Bibliotecas del CSIC, que cuenta con un portal de editores de libros electrónicos (figura 1). También en la Biblioteca virtual soportada por
Metalib, al filtrar por "tipo de recurso", "portal de libros" (figura 2).

http://bibliotecas.csic.es/cbic.html

Pero la entrada más importante es la que proporciona el opac. Tanto las bases de datos, como los libros y las revistas, impresas o electrónicas, se incluyen en el catálogo colectivo Cirbic. Cualquier búsqueda se puede acotar a que sea sólo en el catálogo digital o específicamente en formato de libro electrónico y así acceder al registro concreto (figura 3).

\section{Uso de libros electrónicos en el CSIC en el período 2004-2009}

Aun teniendo una historia tan corta en lo que se refiere a los libros electrónicos, es muy relevante hacer el seguimiento de estos años

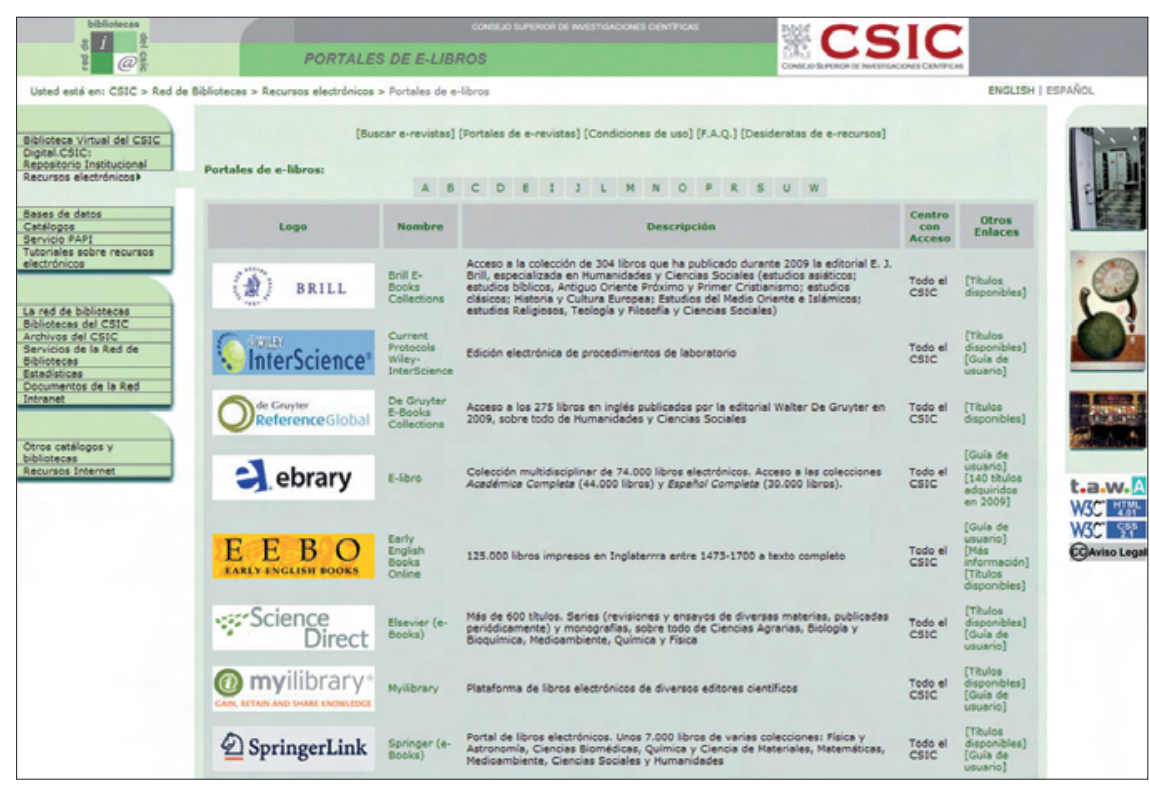

Figura 1. Portales de ebooks

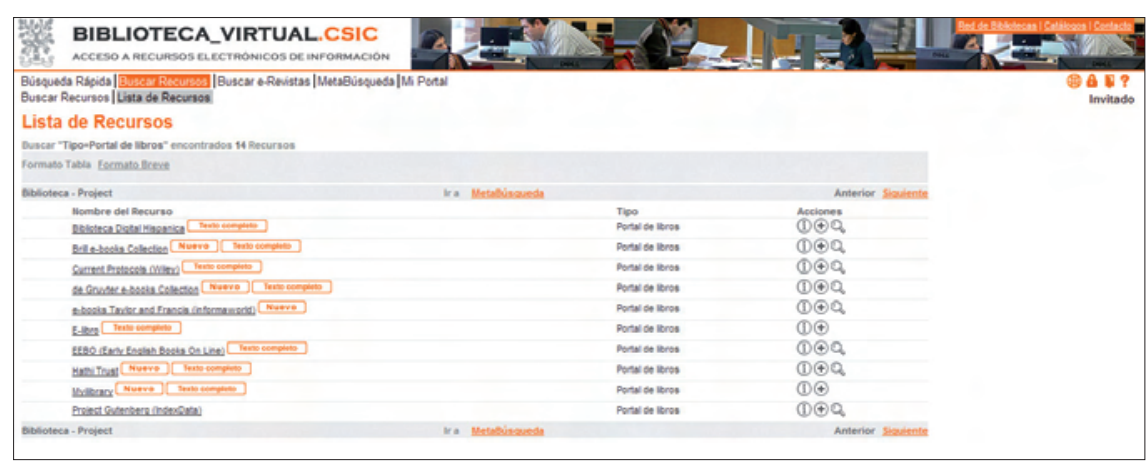

Figura 2. Biblioteca virtual del CSIC 
CATÁLOGO_DIGTTALCSIC

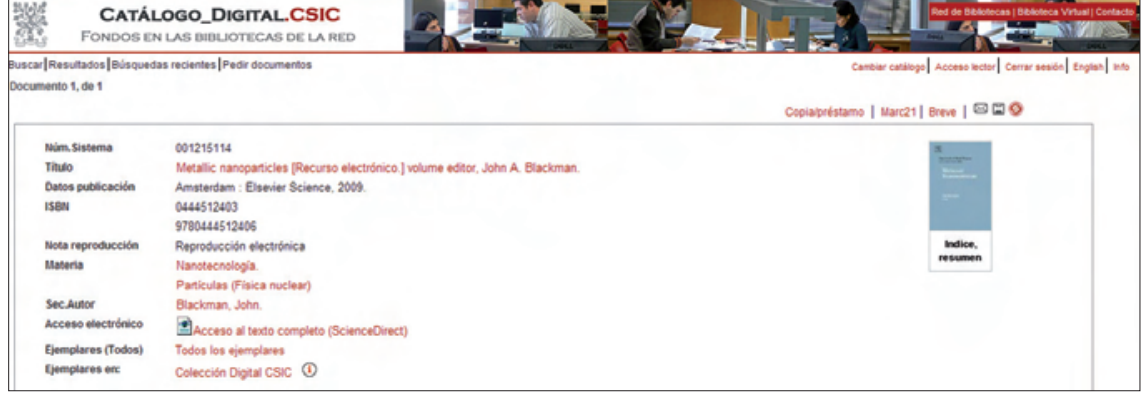

Figura 3. Ejemplo de registro. Catálogo colectivo Cirbic

para descubrir que el uso se ha incrementado a medida que la colección ha crecido y que los libros se han introducido en el catálogo. El despegue del uso de los ebooks no es tan espectacular como fue el de las revistas porque no ha habido suficiente oferta de contenidos.

Los datos que se presentan (tabla 2 y figura 4), además, deben interpretarse con la cautela a la que obliga la falta de uniformidad de parámetros de medida entre los editores de libros electrónicos. En el caso de las revistas hace varios años que la mayoría de los editores se han adherido al sistema Counter, y es posible comparar datos entre ellos y evaluar usos en las propias bibliotecas, porque todos hablamos de "artículos descargados por título". Sin embargo, en el caso de los libros, en 2009 sólo 23 editores se habían comprometido al cumplimiento de Counter code of practice for books and reference works. Esto significa que sólo recientemente, y en pocos editores, los datos de uso de ebooks son homogéneos bajo el parámetro de "capítulos descargados por título".
Hay que observar estos datos, como sucede con las revistas, sabiendo que algunos editores nos devuelven informes de uso en los que se han contado libros, capítulos o partes del libro que son open access, o que han estado en períodos

\section{"Los libros electrónicos que más éxito han tenido son de las materias que tienen mayor uso en las revistas"}

de prueba. No son adquisiciones de la institución, pero registran uso del $C S I C$, y son útiles para mostrar los intereses de los usuarios, aunque estos libros deben excluirse cuando se trata de hacer relaciones de uso/ coste.

Se puede relacionar esta evolución de uso con el momento en que las colecciones se van adquiriendo, y las características de sus informes de uso: Elsevier, en 2005, sólo Book Series. En 2006 y 2007 también Reference work. En 2008 y 2009 también ebooks. En 2007 Springer regaló la colección de arquitectura, pero como no es materia de investigación en el $C S I C$ no tiene mucho éxito. $E E B O$ informa sólo de documentos/títulos. Se trata de obras completas escaneadas, no tiene un posible contador de capítulos. E-libro se adhirió a Counter en 2007, y los datos de 2008 y 2009 sí son capítulos descargados. Hasta entonces informaba sólo de títulos.

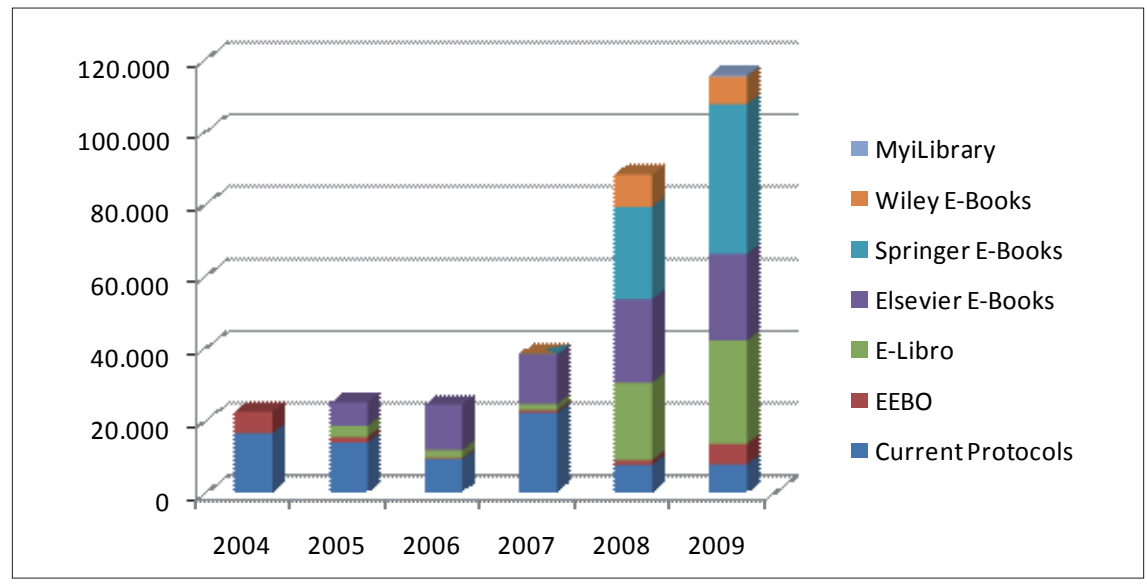

Figura 4. Descargas de capítulos de ebooks

\begin{tabular}{|l|r|r|r|r|r|r|}
\hline \multicolumn{1}{|c|}{ Plataforma } & $\mathbf{2 0 0 4}$ & $\mathbf{2 0 0 5}$ & \multicolumn{1}{c|}{$\mathbf{2 0 0 6}$} & \multicolumn{1}{c|}{$\mathbf{2 0 0 7}$} & \multicolumn{1}{c|}{$\mathbf{2 0 0 8}$} & \multicolumn{1}{c|}{$\mathbf{2 0 0 9}$} \\
\hline Current protocols & 16.407 & 13.871 & 9.215 & 22.073 & 7.639 & 7.756 \\
\hline EEBO & 5.944 & 1.410 & 306 & 678 & 1.305 & 5.698 \\
\hline E-libro & & 3.170 & 2.298 & 1.834 & 21.491 & 28.611 \\
\hline Elsevier e-books & & 6.456 & 12.486 & 13.628 & 23.111 & 24.074 \\
\hline Springer e-books & & & & 120 & 25.590 & 41.424 \\
\hline Wiley e-books & & & & & 8.866 & 7.640 \\
\hline MyiLibrary & & & & & & 420 \\
\hline Total & 22.351 & 24.907 & 24.305 & 38.333 & 88.002 & 115.623 \\
\hline
\end{tabular}

Tabla 2. Descargas de capítulos de ebooks
La colección de revistas electrónicas suscrita por el CSIC se acerca a los 9.000 títulos, y en 2009 ha superado los 2.500 .000 de artículos descargados. Nada que ver con el uso de los libros electrónicos, que nunca alcanzarán niveles semejantes. Además el uso en revistas se concentra en $n$ publicaciones de prestigio, mientras el resto se usa mucho menos. La consulta de ebo- 
oks, sin embargo, se dispersa por toda la colección disponible. Una revista científica tiene asegurado un éxito casi eterno cuando gana prestigio. Los libros, sin embargo, tienen un número de seguidores más corto, menor vigencia en el tiempo, y deben ganarse un espacio en cada ocasión que se publican.

Los libros electrónicos que más éxito han tenido tratan sobre las materias que también tienen mayor uso en las revistas. Las colecciones relacionadas con las ciencias de la vida y con la química son las más consultadas, sin embargo, a la hora de contribuir a la compra de libros electrónicos para la Red, son las bibliotecas de ciencias sociales y humanidades las que más se han comprometido.

\section{"La colección de revistas-e se acerca a los 9.000 títulos, y en 2009 ha superado los 2.500 .000 artículos descargados"}

\section{Inversión en libros electrónicos en 2009}

Por lo que respecta al presupuesto de monografías, en el CSIC no llega a ser ni un $10 \%$ del total que se invierte en el conjunto de recursos de información. Casi el 90\% se lo llevan las revistas, repartido entre las impresas y las digitales. También en las bibliotecas universitarias el presupuesto de revistas es superior al de libros, pero la diferencia es menor que en el CSIC. La razón hay que buscarla en que las fuentes para la información científica son prioritariamente las publicaciones periódicas, y que en esta institución no hay docencia que justifique la compra de manuales o libros de texto para estudiantes.

La apuesta que las bibliotecas del CSIC han hecho por el libro electrónico, mediante estas compras cooperativas organizadas por la $U C B$, ha sido notable. En 2009 han destinado un $22 \%$ de sus presupuestos propios al libro electrónico, y un $78 \%$ al impreso.

\section{"En 2009, las bibliotecas del CSIC destinaron un $22 \%$ de sus presupuestos al libro electrónico y un 78\% al impreso"}

Las bibliotecas del área de humanidades y ciencias sociales y las de química han sido las que más han contribuido en esta adquisición de 2009, que se ha repartido como se indica en la tabla 3 , complementada por la figura 5 .

Estos datos hay que ponerlos en relación con el presupuesto individual que para monografías recibe cada centro. En el caso de las bibliotecas de humanidades y ciencias sociales es significativamente superior al del resto de las áreas de investigación (figura 6).

Aparte de esta inversión en la campaña cooperativa, la Red de Bibliotecas del CSIC sigue reno-

\begin{tabular}{|l|c|}
\hline \multicolumn{1}{|c|}{ Áreas } & $\begin{array}{c}\text { ebooks } \\
\%\end{array}$ \\
\hline $\begin{array}{l}\text { Humanidades y ciencias } \\
\text { sociales }\end{array}$ & 33 \\
\hline $\begin{array}{l}\text { Ciencia y tecnologías } \\
\text { químicas }\end{array}$ & 16 \\
\hline $\begin{array}{l}\text { Unidad de Coordinación } \\
\text { de Bibliotecas }\end{array}$ & 13 \\
\hline Recursos naturales & 9 \\
\hline $\begin{array}{l}\text { Ciencia y tecnologías } \\
\text { físicas }\end{array}$ & 9 \\
\hline $\begin{array}{l}\text { Ciencia y tecnologías de } \\
\text { materiales }\end{array}$ & 7 \\
\hline Biología y biomedicina & 5 \\
\hline Ciencias agrarias & 4 \\
\hline $\begin{array}{l}\text { Ciencia y tecnologías de } \\
\text { alimentos }\end{array}$ & 100 \\
\hline Total & \\
\hline
\end{tabular}

Tabla 3. Distribución de la inversión en ebooks por áreas

vando anualmente las colecciones de libros electrónicos que contrató en 2004: Elsevier book series, Reference works, E-libro y Current protocols.

\section{EI futuro}

La preferencia de la Red de Bibliotecas del CSIC por los libros electrónicos se va a mantener, con el objetivo de ampliar los contenidos e incorporar nuevas editoriales a la colección común. La $U C B$, que gestiona la Biblioteca virtual, vela especialmente para que no haya desequilibrios entre las áreas científicas. El recurso "libro electróni-

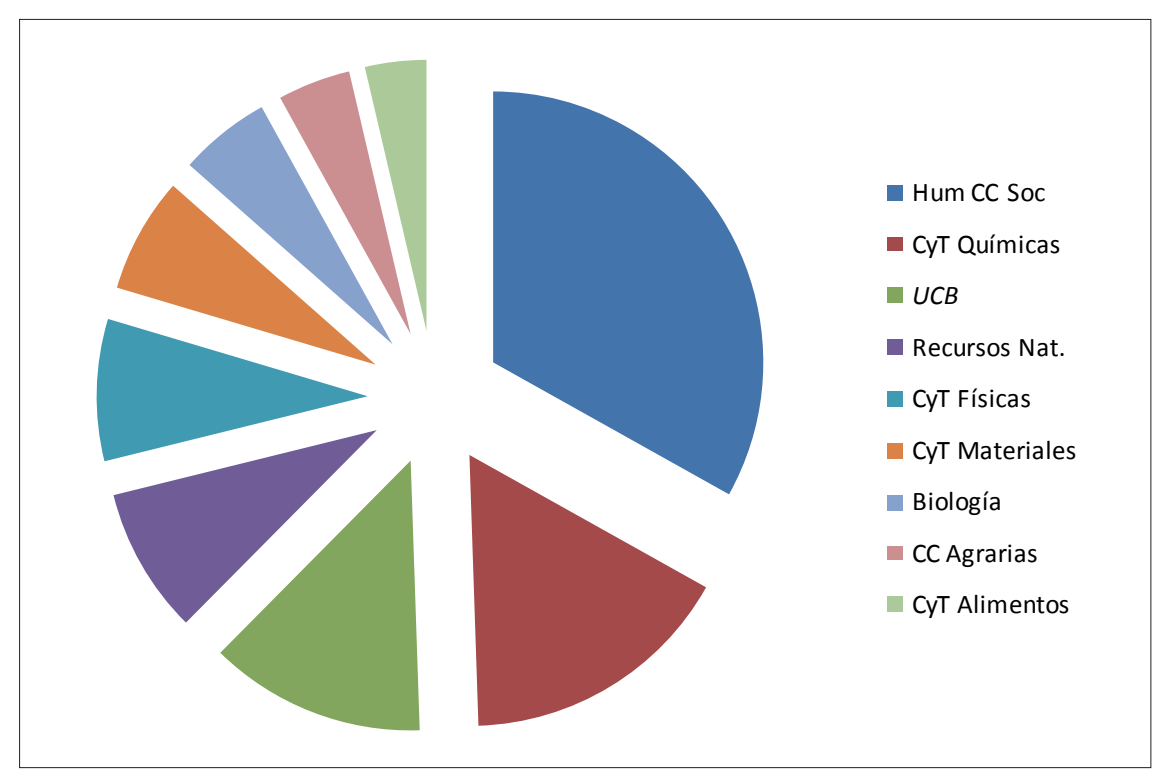

Figura 5. Distribución de la inversión en ebooks por áreas 


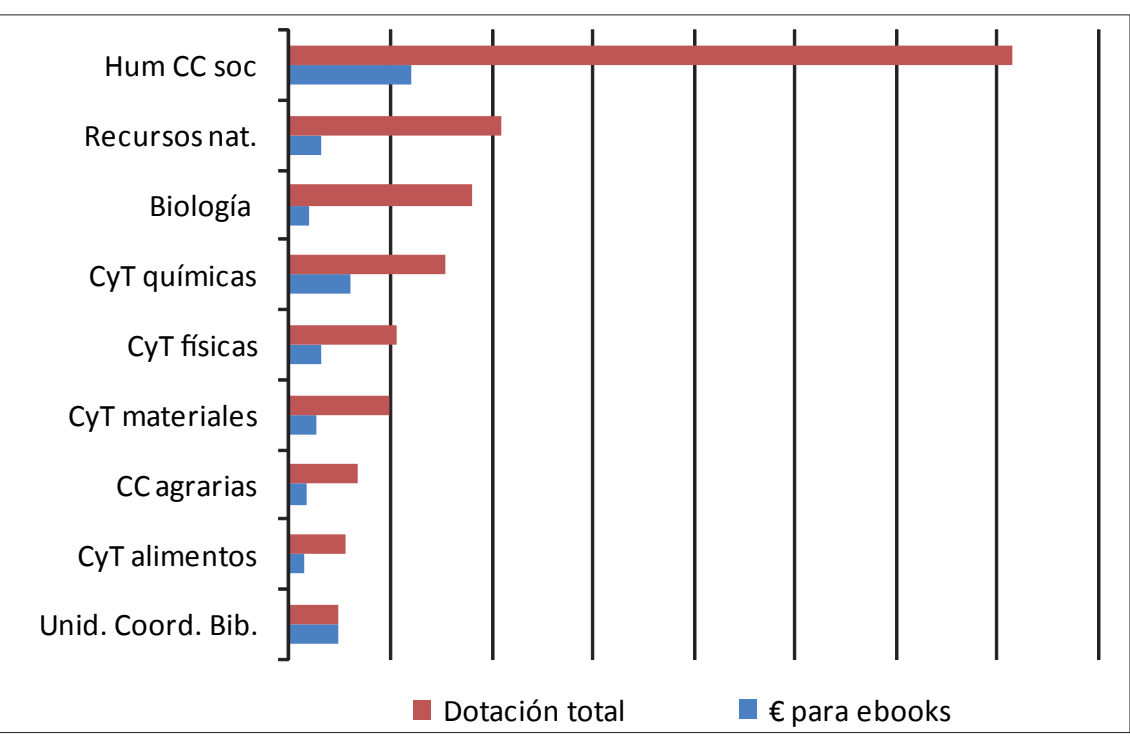

Figura 6. Comparación entre el presupuesto total y el usado para ebooks en las diferentes áreas del CSIC

co" está en el CSIC en una fase de consolidación y es necesario seguir aumentando la oferta para que los usuarios sigan encontrando respuestas digitales a sus búsquedas de monografías (intencionadas, o por serendipity). En este sentido, desde la $U C B$ se tomará en cuenta de modo prioritario la compra de colecciones extensas. Los editores parecen estar optando por diseñar ofertas de big deal, como hicieron con las revistas, aunque siempre está la opción de una compra más individualizada.

\section{"La UCB, que gestiona} la Biblioteca virtual, vela especialmente para que no haya desequilibrios entre las áreas científicas"

Se trabajará para mejorar las formas de acceso a los libros electrónicos, tanto desde las herramientas que ahora mismo sostienen la Biblioteca virtual, como desde el catálogo. La próxima versión del

\section{SFX}

SFX (una abreviatura inventada) es un software que facilita la creación de enlaces de hipertexto dentro de un opac. Fue desarrollado en 1999 por Herbert Van de Sompel en la Universidad de Gante, Bélgica, en colaboración con colegas de Los Álamos National Laboratory, EUA. El producto fue adquirido por Ex Libris, una compañía con sede en Israel que fabrica el software de automatización de bibliotecas Aleph. Ex Libris ha integrado SFX en su otro producto Metalib, un metabuscador o buscador simultáneo en varias bases de datos y catálogos.

Se trata del primer producto de OpenURL o link resolver y su uso está tan generalizado que ambos nombres se confunden.

Los OpenURL se materializan en urls muy largos que contienen en una sola ristra todos los datos de una petición determinada (revista+títulodel-artículo+autor+año+etc.) dirigida a una base de datos preprogramada por cada biblioteca, según sus adquisiciones, donde se resuelve la petición. Un usuario hace clic en tal url y el sistema le redirige -por ejemplo- al pdf del artículo deseado al que la biblioteca tiene derecho de acceso. 
gan mejores precios, cumplan con los requisitos (acceso para todo el CSIC, registros marc, estadísticas Counter, etc.), o respondan a desideratas manifestadas por la comunidad de usuarios a través de las bibliotecas de la Red.

Pero el modelo de compra cooperativa, vigente en estos años 2007-2009 va a cambiar. Como ha sucedido en todas las bibliotecas de nuestro entorno, también en el CSIC se ha producido un recorte en los presupuestos. El de monografías ha disminuido a menos de la mitad del que se tuvo anteriormente. Y, ahora sí, se ha centralizado en la $U C B$, con el mandato institucional de que el destino de este presupuesto sea para libros electrónicos de la colección digital común. Aunque la reducción es una mala noticia, a la larga el resultado final será que la inversión del CSIC en libros electrónicos, y la colección disponible en la Biblioteca virtual común para todos se incrementará significativamente.

\section{Bibliografia}

Alvite-Díez, Luisa; Rodríguez-Bravo, Blanca "E-books in Spanish academic libraries". The electronic library, 2009, v. 27, n. 1, pp. 86-95.

Bucknell, Terry. "The 'big deal' approach to acquiring e-books: a usage-based study". Serials, 2010, v. 23, n. 2, julio, pp. 126-134.

Cordón-García, José-Antonio; Alonso-Arévalo, Julio; Martín-Rodero, Helena. "Los libros electrónicos: la tercera ola de la revolución digital". Anales de documentación, 2010, v. 13, pp. 53-80.
Pérez-Arranz, Fernando; Moscoso, Purificación. "El libro electrónico y su incidencia en las bibliotecas universitarias y científicas españolas". Revista española de documentación científica, 2007, v. 30, n. 3, jul.-sept., pp. 343-363.

Shepherd, Peter; Woodward, Hazel. "The Counter code of practice for books and reference works - a primer". Serials, 2009, v. 22, n. 3-supplement, Nov., pp. S39-S44.

Thompson, Sarah; Sharp, Steve. "E-books in academic libraries: lessons learned and new challenges". Serials, 2009, v. 22, n. 2, Jul., pp. 136-140.

Mercedes Baquero-Arribas. Unidad de Coordinación de Bibliotecas del CSIC.

C/ Joaquín Costa, 22, $3^{a}$ planta, despacho 311.

28002 Madrid.

mbaquero@bib.csic.es

http://bibliotecas.csic.es

\section{Tu futuro es ahora}

Másters y Postgrados IDEC-UNIVERSITAT POMPEU FABRA creemos en ti

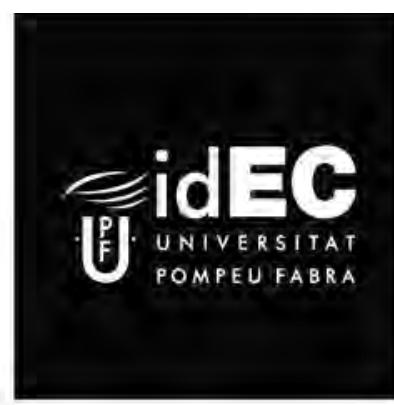

\section{Máster en Documentación Digital}

Máster y Programas de postgrado online

Arquitectura de la información en sitios web - Organización y gestión de la información - Buscadores y posicionamiento web - Documentación audiovisual - Web social - Archivística digital - Gestión de contenidos y gestión del conocimiento - Bibliotecas digitales - Usabilidad y accesibilidad.

Dirección: Cristòfol Rovira y Lluís Codina

Calendario: del 4 de octubre de 2010 al 30 de junio de 2011

www.documentaciondigital.org

\section{Máster en Buscadores: Marketing y Posicionamiento} Máster y Programas de postgrado online

Fundamentos en buscadores - Posicionamiento en Buscadores - SEO - Marketing en Buscadores - SEM

- Analítica web - SEO y SEM en la web social - Vigilancia tecnológica y buscadores especializados.

Dirección: Cristỏfol Rovira, Mari Carmen Marcos y Lluis Codina

Calendario: del 11 de noviembre de 2010 al 22 de junio de 2011

www.masterenbuscadores.com

\section{Colaboran:}

Observatorio de Comunicación Cientifica - UPF Instituto Universitario de Lingüistica Aplicada . UPF DOC 6 Consultores de Recursos de Informaciôn
Infórmate:

IDEC-Universitat Pompeu Fabra www.idec.upf.edu• info@idec.upf.edu

Tel.: +34 $935421850 \cdot$ Balmes, 132-134 • o8008 Barcelona 